\title{
Intestinal parasites of children and adults in a remote Aboriginal community of the Northern Territory, Australia, 1994-1996
}

\author{
Jennifer Shield, ${ }^{a}$ Kieran Aland, ${ }^{b}$ Thérèse Kearns, ${ }^{c}$ Glenda Gongdjalk, ${ }^{d}$ Deborah Holt, ${ }^{c}$ Bart Curriece and Paul Prociv ${ }^{f}$ \\ Correspondence to Jennifer Shield (e-mail: J.Shield@latrobe.edu.au).
}

Introduction: Parasitic infections can adversely impact health, nutritional status and educational attainment. This study investigated hookworm and other intestinal parasites in an Aboriginal community in Australia from 1994 to 1996.

Methods: Seven surveys for intestinal parasites were conducted by a quantitative formol-ether method on faecal samples. Serological testing was conducted for Strongyloides stercoralis and Toxocara canis IgG by enzyme-linked immunosorbent assays.

Results: Of the 314 participants, infections were as follows: Trichuris trichiura (86\%); hookworm, predominantly Ancylostoma duodenale (36\%); Entamoeba spp. (E. histolytica complex [E. histolytica, E. dispar and E. moskovski], E. coli and E. hartmanni) (25\%); S. stercoralis (19\%); Rodentolepis nana (16\%); and Giardia duodenalis (10\%). Serological diagnosis for 29 individuals showed that 28\% were positive for S. stercoralis and $21 \%$ for T. canis. There was a decrease in the proportion positive for hookworm over the two-year period but not for the other parasite species. The presence of hookworm, T. trichiura and Entamoeba spp. was significantly greater in 5-14 year olds $(n=87)$ than in 0-4 year olds $(n=41)$, while the presence of S. stercoralis, $R$. nana, G. duodenalis and Entamoeba spp. in 5-14 year olds was significantly greater than $15-69$ year olds $(n=91)$.

Discussion: Faecal testing indicated a very high prevalence of intestinal parasites, especially in schoolchildren. The decrease in percentage positive for hookworm over the two years was likely due to the albendazole deworming programme, and recent evidence indicates that the prevalence of hookworm is now low. However there was no sustained decrease in percentage positive for the other parasite species.

$\mathrm{P}$ arasitic infections adversely affect health. Heavy infections with helminths cause clinical disease including anaemia, diarrhoea and malabsorption of nutrients. ${ }^{1-5}$ Moderate or light infections cause morbidity by adversely affecting nutritional status ${ }^{6}$ and affect educational outcomes by impairing cognitive processes. ${ }^{7}$ Trichuris trichiura infection has been associated with poor immunogenicity from BCG vaccination. ${ }^{8}$

Several intestinal parasite species occur in Australia with a high prevalence in rural and remote Aboriginal communities. Hookworm (predominantly Ancylostoma duodenale) occurs mainly north of $22^{\circ} \mathrm{S}$ particularly near the coast. ${ }^{9-12}$ Whipworm (T. trichiura) is rare in Western Australia, ${ }^{9}$ more common in Queensland $^{1}$ and also occurs in the Northern Territory (NT). ${ }^{10,13,14}$ Strongyloides stercoralis is more widespread, occurring throughout the northern two thirds of Australia (Jennifer Shield, La Trobe University, unpublished collated data). Dwarf tapeworm (Rodentolepis nana [= Hymenolepis nana $]$ ) and the protozoan Giardia duodenalis are common in Aboriginal communities throughout Western Australia, ${ }^{9}$ Queensland ${ }^{1}$ and the NT. ${ }^{10,13}$ Entamoeba spp. occur throughout Australia. ${ }^{1,15,16}$ Among the pathogenic species of intestinal protozoa, Entamoeba histolytica is the second leading cause of death globally after malaria, ${ }^{15}$ and G. duodenalis causes chronic diarrhoea, malabsorption and weight loss and contributes to stunting and cognitive impairment in children. ${ }^{17}$

In the past, morbidity of parasitic infections was assessed on a species-by-species basis. Current evidence, however, suggests that when a person is

\footnotetext{
La Trobe University, Bendigo, Victoria, Australia.

Queensland Museum, Brisbane, Queensland, Australia

Menzies School of Health Research, Darwin, Northern Territory, Australia.

Ngalkanbuy Health Centre, Galiwin'ku, Northern Territory, Australia

Royal Darwin Hospital, Darwin, Northern Territory, Australia.

University of Queensland, Brisbane, Queensland, Australia.

Submitted: 14 January 2015; Published: 6 March 2015

doi: 10.5365/wpsar.2015.6.1.008
} 
infected with two or more parasite species, there is an additive or multiplicative impact on nutrition and organ pathology. ${ }^{18}$

There is a shortage of published surveys of intestinal parasites in Australia. This paper documents the prevalence of intestinal parasites in a remote Aboriginal community in Australia from 1994 to 1996. Historical data such as these are rare and are of intrinsic value. In addition, they provided baseline data with which to compare the current situation, enabling the evaluation of deworming strategies.

\section{METHODS}

\section{Subjects and location}

This study was conducted in a remote township, in north-eastern Arnhem Land between July 1994 and October 1996. Most residents belong to the Yolngu group of Aboriginal Australians.

\section{Collection of faecal samples}

Each household was visited by the parasitologist and health educator. Information about hookworm infection, transmission and diagnosis was given in the local language using prepared illustrations, and then a faecal sample was requested. Each person was given a labelled rectangular plastic container with a snap-on lid and a brown paper bag. The containers were collected the following morning. Sampling occurred during seven time periods: July 1994 (1 to 18 July), October 1994 (30 September to 19 October), December 1994 (8 to 19 December), May 1995 (5 to 22 May), August 1995 (4 to 19 August), December 1995 (20 November to 19 December) and October 1996 (exact dates not recorded). Collections in November and December were during the wet season; the other collections were during the dry season.

\section{Examination of faecal samples}

In the field laboratory, a direct saline wet preparation of the faecal specimen was examined microscopically as soon as possible, and a $0.91 \mathrm{~mL}$ aliquot of faeces was preserved in $5-10 \mathrm{~mL} 4 \%$ formaldehyde for later examination by a quantitative formol-ether count method. Hookworm and T. trichiura eggs were counted and the presence of $S$. stercoralis larvae, $R$. nana eggs, G. duodenalis, Entamoeba spp. and other protozoa cysts or trophozoites were noted. The number of eggs per $\mathrm{mL}$ faeces was calculated by multiplying the count by a factor (total volume of faecal suspension/[volume of the faecal sample $x$ volume of sample counted]). The size of the multiplication factor varied between 10 and 42 . The counts were adjusted for faecal consistency using the Stoll correction factors. ${ }^{19}$

\section{Determination of hookworm species}

Vermiculite cultures of 10 faecal samples with a substantial number of eggs in the direct smear were prepared to determine the species of hookworm based on the morphology of the cultured filariform larvae. ${ }^{20}$

Adult hookworms were collected from the digestive system on autopsy of stray dogs. Twelve adult worms were measured and examined in detail to determine the species.

\section{Serology (specific serum immunoglobulin G [IgG] tests)}

Venous blood was collected from 39 adults and children as part of their normal routine testing and IgG levels against Strongyloides and Toxocara were measured using enzyme-linked immunosorbent assay. The Strongyloides assay used S. ratti $\mathrm{L} 3$ as the antigen and had an estimated sensitivity of $93 \%$ and specificity of $95 \% .{ }^{21}$ The Toxocara assay used $T$. canis excretory/secretory antigen as the antigen and had an estimated sensitivity of $91 \%$ and specificity of $86 \% .^{22}$

\section{Statistical analysis}

A parasitic infection was defined as specimens positive by the quantitative formol-ether method only. Survey results included participants who were present at more than one survey; only the first examination was included. Geometric means of egg counts of infected people were calculated using logarithm to base e (In) transformed data and egg counts recovered by antilogarithm. When the egg counts for $A$. duodenale and $T$. trichiura were grouped using a logarithmic scale, the plotted frequencies had a normal distribution, indicating that statistical methods applicable to normal distributions were valid when performed on logarithmically transformed egg count 
data. Intensity of infection was classified according to World Health Organization categories ${ }^{23}$ (assuming that eggs/mL is approximately equivalent to eggs/g faeces).

The difference of two independent proportions was compared by calculation of the $Z$ ratio, taking the two-tail value for $P$ (probability). Where the number infected or the number negative was too small for this test, the Fisher's Exact Test was used. Ln transformed mean egg counts were compared using a two-tailed $t$-test for independent samples and unequal variances. Frequency data were analysed using Yates' chi-square test. A $P$-value of $<0.05$ was considered significant. All statistical calculations were performed by the online calculators provided by the VassarStats Web Site for Statistical Calculations (http://www.vassarstats. net/). Graphs were drawn using Excel.

\section{Ethics statement}

Ethical approval for this study was obtained from the Human Research Ethics Committee of the NT Department of Health and Menzies School of Health Research (Registration No. EC00153), approval number 94/19. Everyone who tested positive for parasites by the field smear was notified and asked to go to the health centre for treatment.

\section{RESULTS}

\section{Intestinal parasites: combined faecal count data from all surveys}

Of the 314 participants, the overall percentage positive for intestinal parasites was $89 \%$ and for one or more helminth species was $88 \%$. The most common parasite species was T. trichiura (86\% positive) followed by hookworm (36\%), Entamoeba spp. [E. histolytica complex: E. coli and E. hartmanni] (29\%); S. stercoralis (19\%), R. nana (16\%) and G. duodenalis (10\%). Enterobius vermicularis, Chilomastix mesnilii, Retortamonas intestinalis and Blastocystis spp. were present in a small number of participants.

The intensity of infection for the 314 participants for hookworm was: negative: 64\%; light (1-1999 eggs/mL faeces): 35\%; moderate (2000-3999 eggs/mL): $1 \%$; and for T. trichiura was: negative: 16\%; light (1-999 eggs/mL): 50\%; moderate (1000-9999 eggs/mL): $30 \%$; and heavy ( $\geq 10000$ eggs $/ \mathrm{mL}$ ): 4\%.
Serological diagnosis for 39 individuals showed that $28 \%$ were positive and $18 \%$ equivocal for S. stercoralis, and $21 \%$ were positive and $5 \%$ equivocal for T. canis. The median age for the 21 persons for whom ages were known was 32 years (IQR 24 to 41 ).

\section{Comparison of the seven surveys}

There were 55 (18\%) participants represented in more than one survey. There was significant variation in the percentage of participants positive for hookworm and $S$. stercoralis over the study period (Table 1 ). The percentage positive for hookworm was high in October 1994 preceding the wet season, low in August 1995 following the introduction of an albendazole treatment programme and high in December 1995 during the wet season. There was a significant decrease between October 1994 and October 1996 ( $P<0.0002$; Table 1 ). The percentage positive for $S$. stercoralis was high in May 1995, low in August 1995 following the introduction of albendazole, high in December 1995 (wet season) and low in October 1996 (Table 1). There was no significant variation in the percentage of participants positive for $T$. trichiura, R. nana, G. duodenalis or Entamoeba spp.

There was no significant variation in the geometric mean egg counts of positive samples during the seven surveys for hookworm (Table 1). For T. trichiura, the geometric mean of egg counts in December 1995 (2200 eggs $/ \mathrm{mL}$ ) was significantly greater than those of all the other surveys $(P<0.001)$ (Table 1$)$. This result was likely due to markedly increased egg counts in children (0-14 years) at that time.

\section{Comparisons of age groups and sex}

The median age of the 219 participants whose age was known was 11 years (IQR 6 to 29) (Figure 1); 129 children were aged 0-14 years (approximately $37 \%$ of the child population) and 91 adults were aged 15 years and above (approximately $12 \%$ of the adult population). There was a significantly greater percentage positive in schoolchildren (5-14 years) compared with preschool children (0-4 years) for any parasitic infection, hookworm, T. trichiura and Entamoeba spp, and a significantly lower percentage positive in adults ( $\geq 15$ years) compared with schoolchildren for S. stercoralis, R. nana, G. duodenalis and Entamoeba spp (Figure 1). Hookworm, T. trichiura, R. nana and 
Table 1. Number and proportion of participants who tested positive for intestinal parasites by survey, Northern Territory, Australia, July 1994 to October 1996, $n=383$

\begin{tabular}{|c|c|c|c|c|c|c|c|c|c|c|}
\hline \multirow[b]{2}{*}{$\begin{array}{l}\text { Survey } \\
\text { date }\end{array}$} & \multirow{2}{*}{$\begin{array}{l}\text { No. } \\
\text { people } \\
\text { tested }\end{array}$} & \multirow{2}{*}{$\begin{array}{c}\text { Any parasite } \\
\text { Positive } \\
\text { No. (\%) }\end{array}$} & \multicolumn{2}{|c|}{ Hookworm } & \multicolumn{2}{|c|}{ T. trichiura } & \multirow{2}{*}{$\begin{array}{l}\text { S. stercoralis } \\
\text { Positive } \\
\text { No. (\%) }\end{array}$} & \multirow{2}{*}{$\begin{array}{l}\text { R. nana } \\
\text { Positive } \\
\text { No. (\%) }\end{array}$} & \multicolumn{2}{|c|}{ G. duodenalis Entamoeba spp } \\
\hline & & & $\begin{array}{l}\text { Positive } \\
\text { No. (\%) }\end{array}$ & $\begin{array}{c}\text { Geom mean } \\
(\operatorname{eggs} / \mathrm{mL}) \\
{[\operatorname{Exp}(\mathrm{Cl})]}\end{array}$ & $\begin{array}{l}\text { Positive } \\
\text { No. (\%) }\end{array}$ & $\begin{array}{c}\text { Geom mean } \\
(\operatorname{eggs} / \mathrm{mL}) \\
{[\operatorname{Exp}(\mathrm{CI})]}\end{array}$ & & & $\begin{array}{l}\text { Positive } \\
\text { No. (\%) }\end{array}$ & $\begin{array}{l}\text { Positive } \\
\text { No. (\%) }\end{array}$ \\
\hline \multirow[t]{2}{*}{ Jul 1994} & 62 & 57 (92) & $19(31)$ & 200 & $53(86)$ & 820 & $13(21)$ & $15(24)$ & $10(16)$ & $18(29)$ \\
\hline & & & & [120-350] & & [520-1300] & & & & \\
\hline \multirow[t]{2}{*}{ Oct 1994} & 76 & $73(94)$ & $43(57)^{*}$ & 180 & $68(89)$ & 560 & $13(16)$ & $13(16)$ & $9(11)$ & $22(27)$ \\
\hline & & & & [120-250] & & [390-800] & & & & \\
\hline \multirow[t]{2}{*}{ Dec 1994} & 37 & $31(84)$ & $17(46)$ & 110 & $29(78)$ & 400 & $4(11)$ & $3(8)$ & $3(8)$ & $11(30)$ \\
\hline & & & & [61-190] & & [260-620] & & & & \\
\hline \multirow[t]{2}{*}{ May 1995} & 48 & $44(92)$ & $16(33)$ & 120 & $40(83)$ & 800 & $17(35)^{*}$ & $7(15)$ & $0(0)$ & $10(21)$ \\
\hline & & & & [73-200] & & {$[460-1400]$} & & & & \\
\hline Jun 1995 & & Single-dose alk & sendazole $\mathrm{d}$ & eworming prog & ramme be & gins, replacing & previous progr & mmes usir & ng pyrantel an & bendazole. \\
\hline \multirow[t]{2}{*}{ Aug 1995} & 63 & $57(90)$ & $7(11)^{* \dagger}$ & 130 & $53(84)$ & 520 & $6(19)^{*}$ & $12(19)$ & $4(6)$ & $23(37)$ \\
\hline & & & & [42-420] & & [360-740] & & & & \\
\hline \multirow[t]{2}{*}{ Dec 1995} & 44 & $41(93)$ & $15(34)^{*}$ & 140 & $38(86)$ & $2200^{* \neq}$ & $13(30)^{*}$ & $12(27)$ & $5(11)$ & $17(27)$ \\
\hline & & & & [79-250] & & [1400-3300] & & & & \\
\hline \multirow[t]{2}{*}{ Oct 1996} & 53 & $46(87)$ & $13(25)^{\dagger}$ & 100 & $44(83)$ & 380 & $4(8)^{*}$ & $7(13)$ & $5(9)$ & $21(40)$ \\
\hline & & & & [53-180] & & [230-640] & & & & \\
\hline \multirow[t]{2}{*}{ Total $^{\S}$} & 383 & 349 (91) & 130 (34) & 100 & 325 (85) & 650 & 70 (18) & 69 (18) & $36(9)$ & $122(32)$ \\
\hline & & & & [110-190] & & [550-780] & & & & \\
\hline
\end{tabular}

Exp $(\mathrm{Cl})$, exponentially recovered confidence interval; Geom mean, geometric mean for those infected.

* Significant difference with the previous survey.

$\dagger$ Significant difference with the survey conducted approximately one year earlier.

¥ Significant difference with the geometric means of all other surveys.

$\S$ Some participants were included in more than one survey.

Figure 1. Proportion of intestinal parasites detected among participants by age group* and species, Northern Territory, Australia, July 1994 to October 1996

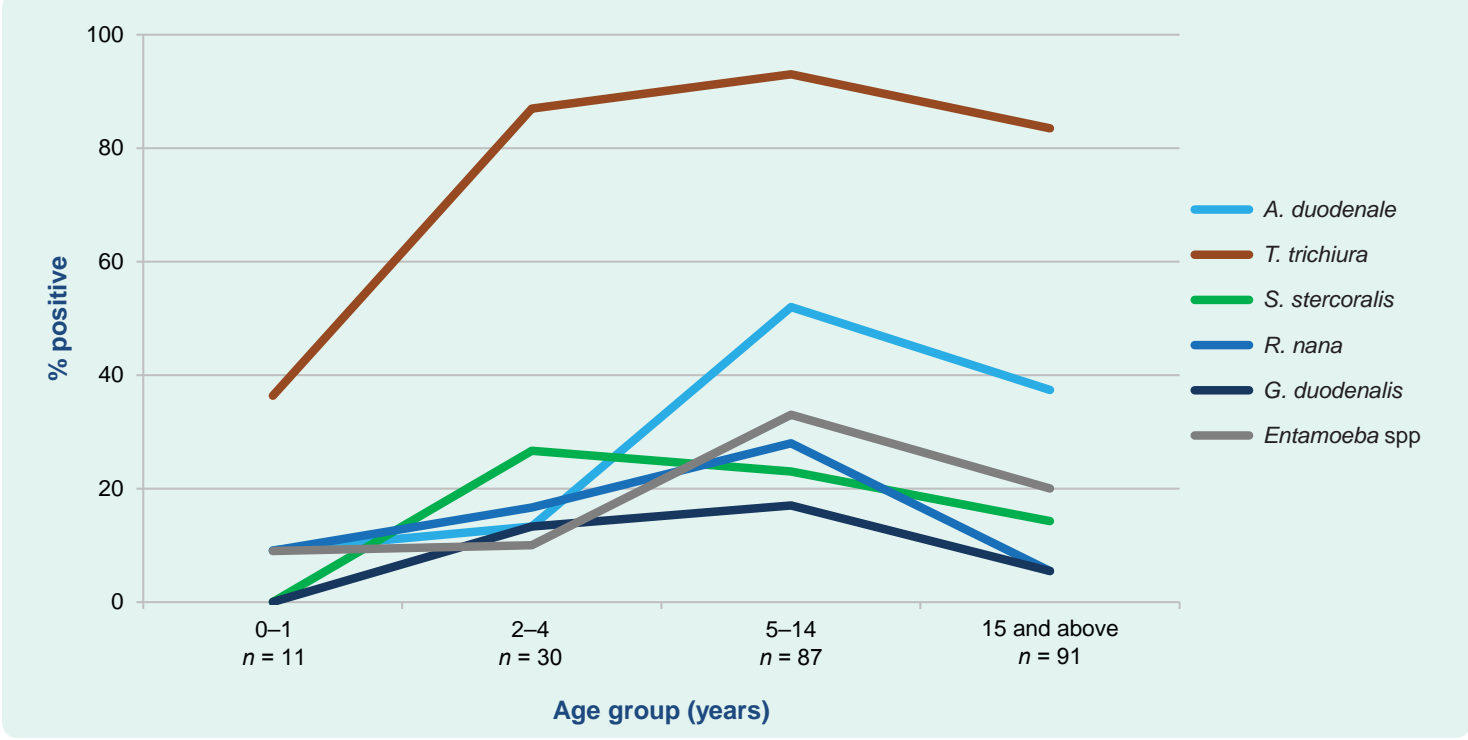

* Age group was available for 219 (70\%) of participants, 0-4 years age group was divided into 0-1 and 2-4. 
Entamoeba spp. were present in 0-1 year old children. All six species were present in 2-4 year old children (Figure 1).

For $T$. trichiura, the geometric mean egg count in infected schoolchildren (1200 eggs $/ \mathrm{mL}$ ) was significantly greater than in both preschool children (400 eggs $/ \mathrm{mL}$ ) and adults $(410$ eggs $/ \mathrm{mL})(P<0.001)$. For hookworm, there was no significant difference between geometric mean egg counts for the age groups.

The sex was known for 100 males and 115 females. The percentage positive in males was significantly greater than in females for hookworm (47\% compared with $30 \% ; P=0.012), T$. trichiura (92\% compared with $79 \% ; P=0.0082$ ) and $G$. duodenalis ( $17 \%$ compared with $6 \% ; P=0.011$ ).

\section{Polyparasitism}

Of the 314 participants, 152 (48\%) were infected with more than one helminth species and 109 (35\%) were infected with one or more helminth species and one or more protozoa species (Figure 2). There was a statistically significant association of helminth infection with protozoa infection $(P=0.0039)$.

\section{Hookworm species}

Infective larvae recovered from vermiculite faecal cultures were all identified as Ancylostoma spp., most likely Ancylostoma duodenale. Adult Ancylostoma collected from dogs were all identified as $A$. caninum.

\section{DISCUSSION}

These results indicate a very high prevalence of infection with one or more intestinal parasite species in this community between 1994 and 1996 despite the deworming programme in children that was conducted from before the study began until the present time. In 1994, the standard treatment was pyrantel $20 \mathrm{mg} / \mathrm{kg}$ as a single dose or mebendazole $100 \mathrm{mg}$ twice daily for three days. From May 1995 albendazole (single dose of $200 \mathrm{mg}$ for children 8 to $<10 \mathrm{~kg}$ body weight and $400 \mathrm{mg}$ for children $\geq 10 \mathrm{~kg}$ ) was the usual anthelmintic treatment during yearly child health checks; for adults and children with suspected or confirmed gastrointestinal helminths, the standard treatment was daily albendazole $400 \mathrm{mg}$ for three days. The parasite species detected by
Figure 2. Proportion of helminth infections with and without protozoa infection, Northern Territory, Australia, July 1994 to October 1996, $(n=314)^{*}$

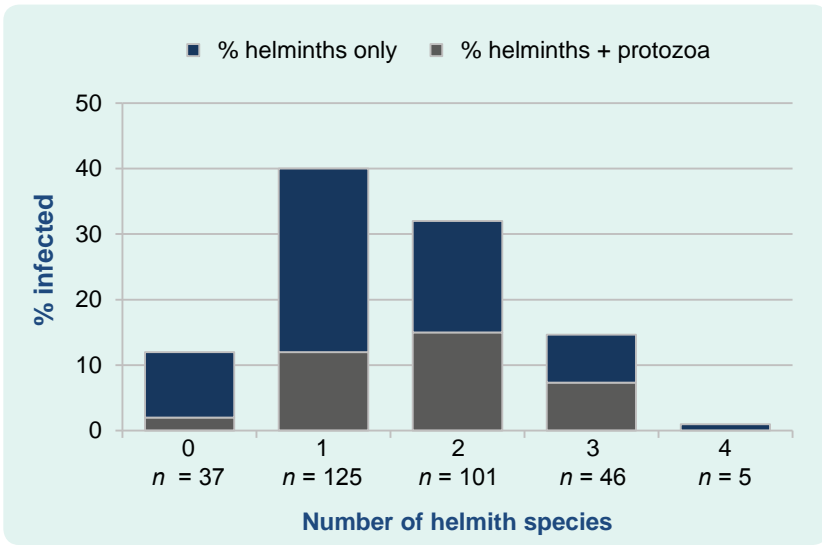

* There was a statistically significant association of helminth infection with protozoa infection $(P=0.0039)$.

faecal testing were the same as in two smaller studies in the same region at about the same time, ${ }^{10,13}$ but the percentages positive in our study were greater. This likely reflects the greater sensitivity of the quantitative formolether method. Even though this method was much more sensitive than direct smears due to the relatively large amount of faeces examined, it likely underestimated the true prevalence; for example, $28 \%$ of a small group ( $n=39$ ) were positive for S. stercoralis by serology compared with $19 \%$ positive by formol-ether count $(n=314)$. A study of a similar method using ethyl acetate instead of ether showed that it was much less sensitive than agar plate culture in detecting S. stercoralis. ${ }^{24}$ A positive test by serology indicates a current infection because if the $S$. stercoralis are eliminated, the antibody levels decrease to negative within three to six months of effective treatment. ${ }^{25,26}$

Although the larvae harvested from the vermiculite cultures were Ancylostoma sp, probably A. duodenale, the presence of Necator americanus cannot be excluded. A. ceylanicum may also be present, as it is present in dogs in Australia ${ }^{27}$ and has also been detected in people. ${ }^{28}$ The observed seasonal variations in the percentage positive for hookworm provide additional evidence that the hookworm was at least predominantly $A$. duodenale. The variation is likely due to larval hypobiosis during the dry season and increased transmission during the wet season. ${ }^{29}$ The lifespan of adult $A$. duodenale is approximately six months. Larvae that enter the body late in the wet season do not develop but remain in the tissues and mature late in the dry season. Treatment 
with pyrantel eradicates adult $A$. duodenale but not the dormant larvae. ${ }^{29}$ The peak in percentage positive in October 1994, late in the dry season, is consistent with this. Increased transmission during the wet season accounts for the high percentage positive for A. duodenale in December 1994 and 1995.

The very high prevalence of infection with two or more intestinal parasites observed in this study is of concern; individuals with multiple infections suffer increased morbidity ${ }^{18}$ due to the differing pathologies caused by the different parasitic species. A greater prevalence of hookworm, T. trichiura and G. duodenalis in males than females has been observed elsewhere. ${ }^{30}$

The greater percentages positive for intestinal parasites in school-aged children compared with other age groups follows patterns elsewhere. ${ }^{18}$ There was an appreciable percentage positive in very young children of 2-4 years at this critical stage in their development. The finding that $R$. nana and $G$. duodenalis infections occur primarily in children under 15 years of age is consistent with findings in Aboriginal communities throughout Western Australia. ${ }^{9}$ The percentage of participants positive for hookworm and T. trichiura in schoolchildren compared with adults were not significantly different, but the infection intensity of T. trichiura in schoolchildren was significantly greater than in adults.

The finding of an appreciable percentage of adult participants positive for hookworm, T. trichiura and S. stercoralis justifies regular anthelmintic treatment of adults as well as children. The lower percentage of adult participants positive for S. stercoralis compared with schoolchildren is of interest since infection with this species can be life-long in the absence of effective treatment. $^{31}$ Single-dose albendazole does not usually eliminate the infection. ${ }^{25}$

The presence of $A$. caninum in dogs raises the possibility of zoonotic infections in humans, which may be asymptomatic or cause eosinophilic enteritis. ${ }^{32}$ Seroepidemiological studies showed it to be common in Queensland in the 1990s. ${ }^{32}$ While eosinophilic enteritis has been recognized in the non-Aboriginal population of the NT, it has not been documented as a clinical issue in the Aboriginal population, ${ }^{33}$ possibly because of the albendazole deworming programme.
T. canis is common in dogs and is found as eggproducing adult worms mainly in pups. Our finding of specific IgG against T. canis in $21 \%$ of people tested indicates a significant level of human exposure to infection (putatively via egg ingestion). By way of comparison, $43 \%$ of another Aboriginal group in far north-eastern Arnhem Land, ${ }^{10} 11.1 \%$ of another NT group and $5.7 \%$ of the general Australian population ${ }^{34}$ were seropositive in the 1990s. The clinical significance of $T$. canis in the Aboriginal population is unknown. The larvae can cause hepatic disease (visceral larva migrans) or eye lesions (ocular larva migrans). ${ }^{19}$ Visceral larva migrans has been occasionally diagnosed in the non-Aboriginal population of the NT, but confirmed cases in the Aboriginal population have not occurred in recent years (Bart Currie, Royal Darwin Hospital, unpublished data).

The variations in S. stercoralis percentage positive are difficult to interpret. Intermittent larval shedding has been documented in this species $^{35}$ and may explain the variations seen. Another possibility is that pyrantel, mebendazole or albendazole treatment was not eradicating infection but causing only a temporary decrease in larval output. Currently, the use of ivermectin is greatly improving the efficacy of treatment. ${ }^{25}$ The effectiveness of community-wide ivermectin treatment to reduce $S$. stercoralis infection is currently being assessed. 26

The results of the October 1996 survey suggest that the albendazole treatment programme was reducing the prevalence of hookworm as the percentage of participants positive for hookworm decreased significantly at a time of year when an increase would be expected from maturation of dormant larvae of $A$. duodenale. This result is also consistent with the eradication of the dormant larvae in those who had received albendazole treatment. Initial treatment with albendazole was likely responsible for the drop in percentage positive for hookworm in August 1995. Recent work indicates that $A$. duodenale is now well controlled, ${ }^{36,37}$ but a high proportion of the children tested were positive for $T$. trichiura and $R$. nana. ${ }^{37}$ A recent review of laboratory data indicated that the detection rates of $T$. trichiura in the NT have substantially decreased over the last decade. ${ }^{14}$ Singledose albendazole is much less effective at eliminating T. trichiura in children than in adults ${ }^{38}$ and is not effective against $R$. nana. ${ }^{39}$ Our results are consistent 
with this. The efficacy of albendazole against $T$. trichiura, $R$. nana and G. duodenalis is improved if given daily for three days. ${ }^{38,39}$

It is not possible to conclude whether the Entamoeba spp. observed were of public health significance. Determination of the extent of the recognized pathogens E. histolytica and E. moshkovski present in participants would require molecular faecal testing that is available now but not at the time of the study.

A factor that probably contributes to the high prevalence of parasites is the large number of people per house, ${ }^{40}$ putting pressure on food preparation, washing and sanitary facilities. ${ }^{41}$ Recently, there has been an attempt to address this issue with the addition of new and renovated houses.

There is an urgent need for a more holistic approach to the control of the remaining parasites, combining health promotion and adequate treatment. A key ingredient is community empowerment through respectful adult community education.

In this study, the samples were not randomly selected, sample sizes were small and largely made up of newly recruited participants. There was variation in the proportion of children (45-70\%) and in the maleto-female ratio (0.8 to 1.7) from survey to survey. The pooled values used to compare different age groups, sex and multiple infections did not take into account the seasonal variation in hookworm or S. stercoralis. Documentation of anthelmintic treatment at the individual level depended on health centre records which were incomplete; therefore we examined the effect of the treatment programme on the prevalence of the parasites. There was no attempt to distinguish between new infections and those detected in the previous surveys.

\section{CONCLUSIONS}

In spite of the above limitations, this study highlights the very high prevalence of infection with intestinal parasites, frequently with more than one species, in this remote Aboriginal community in the 1990s, particularly in schoolchildren. The survey data showed that, following the introduction of a yearly single-dose albendazole treatment programme for children, there was a significant reduction in the percentage of participants positive for hookworm but no significant change in the other species of intestinal parasites. This historical data adds to our limited knowledge of parasitic infections in the 1990s and provides a base line for comparison with the current situation, enabling the evaluation of deworming strategies.

\section{Conflicts of interest}

None declared.

\section{Funding}

This work was funded by a National Health and Medical Research Council grant through the Public Health Research and Development Committee to the Ngalkanbuy Health Centre at Galiwin'ku for hookworm research. Kieran Aland was supported by a Commonwealth Postgraduate Scholarship at the University of Queensland.

\section{Acknowledgements}

The authors gratefully acknowledge the people of the Galiwin'ku community who provided faecal and blood samples to enable this study to take place; John Walker and Rogan Lee, Pathology West, Institute of Clinical Pathology and Medical Research, Westmead, Sydney, NSW, who provided the serology results and details of the method used, respectively; and David Shield, who was the statistical consultant for the data analysis.

\section{References:}

1. Jose DG, Welch JS. Growth retardation, anaemia and infection, with malabsorption and infestation of the bowel. The syndrome of protein-calorie malnutrition in Australian Aboriginal children. The Medical Journal of Australia, 1970, 1:349-356. pmid:5439141

2. Layrisse $\mathbf{M}$, Roche $M$. The relationship between anemia and hookworm infection. Results of surveys of rural Venezuelan population. American Journal of Hygiene, 1964, 79:279-301. pmid: 14159948

3. Lucas SB et al. Aberrant form of Hymenolepis nana: possible opportunistic infection in immunosuppressed patients. Lancet, 1979, 2:1372-1373. doi:10.1016/S0140-6736(79)92859-9 pmid:92715

4. Cooper ES, Bundy DA, Henry FJ. Chronic dysentery, stunting, and whipworm infestation. Lancet, 1986, 2:280-281. doi:10.1016/ S0140-6736(86)92093-3 pmid:2874297

5. Speare R. Fatal strongyloidiasis: lessons from the literature. In: Second National Workshop on Strongyloidiasis. Brisbane, Queensland, 2003 (http://www.jcu.edu.au/school/phtm/PHTM/ss/ acrrm-cd/CD-Index.pdf, accessed 11 February 2015).

6. Stephenson LS et al. Treatment with a single dose of albendazole improves growth of Kenyan schoolchildren with hookworm, Trichuris trichiura, and Ascaris lumbricoides infections. The American Journal of Tropical Medicine and Hygiene, 1989, 41:78-87. pmid:2764230 
7. Ezeamama AE et al. Helminth infection and cognitive impairment among Filipino children. The American Journal of Tropical Medicine and Hygiene, 2005, 72:540-548. pmid:15891127

8. Elias D et al. Poor immunogenicity of BCG in helminth infected population is associated with increased in vitro TGF-beta production. Vaccine, 2008, 26:3897-3902. doi:10.1016/j. vaccine. 2008.04 .083 pmid: 18554755

9. Jones HI. Intestinal parasite infections in Western Australian Aborigines. The Medical Journal of Australia, 1980, 2:375-380. pmid:7453610

10. Flannery G, White N. Immunological parameters in northeast Arnhem Land Aborigines: consequences of changing settlement patterns and lifestyles. In: Schell M, Bilsborough A, editors. Urban Ecology and Health in the Third World. Society for the Study of Human Biology Symposium Series. Melbourne, Cambridge University Press, 1993, pp. 202-220.

11. Prociv P, Luke RA. The changing epidemiology of human hookworm infection in Australia. The Medical Journal of Australia, 1995 162:150-154. pmid:7854229

12. Hopkins RM et al. The prevalence of hookworm infection, iron deficiency and anaemia in an aboriginal community in north-west Australia. The Medical Journal of Australia, 1997, 166:241244. pmid: 9076267

13. Fryar D, Hagan S. Pilot screening program for intestinal parasites and anaemia in adults in a Top End Aboriginal community. Northern Territory Communicable Diseases Bulletin, 1997, 4:20-21.

14. Crowe AL et al. Decreasing prevalence of Trichuris trichiura (whipworm) in the Northern Territory from 2002 to 2012. The Medical Journal of Australia, 2014, 200:286-289. doi: 10.5694/mja13.00141 pmid:24641155

15. van $\mathrm{Hal}$ SJ et al. Amoebiasis: current status in Australia. The Medical Journal of Australia, 2007, 186:412-416. pmid:17437396

16. McCarthy JS et al. Endemic invasive amoebiasis in northern Australia. The Medical Journal of Australia, 2002, 177:570. pmid: 12429010

17. Ali SA, Hill DR. Giardia intestinalis. Current Opinion in Infectious Diseases, 2003, 16:453-460. doi:10.1097/00001432200310000-00012 pmid:14501998

18. Drake LJ, Bundy DA. Multiple helminth infections in children: impact and control. Parasitology, 2001, 122 Suppl;S73-81. doi:10.1017/S0031182000017662 pmid:11442199

19. Beaver PC, Jung RC, Cupp EW. Clinical Parasitology. 9th ed. Philadelphia, Lea \& Febiger, 1984.

20. Basic laboratory methods in medical parasitology. Geneva, World Health Organization, 1991.

21. Grove DI. Diagnosis. In: Grove DI, editor. Strongyloidiasis a major roundworm infection of man. London, Taylor and Francis, 1989, pp. 175-197.

22. Jacquier $P$ et al. Immunodiagnosis of toxocarosis in humans: evaluation of a new enzyme-linked immunosorbent assay kit. Journal of Clinical Microbiology, 1991, 29:1831-1835. pmid: 1774303

23. Montresor A et al. Guidelines for the evaluation of soil-transmitted helminthiasis and schistosomiasis at community level: a guide for managers of control programmes. Geneva, World Health Organization, 1998.

24. Intapan PM et al. Comparison of the quantitative formalin ethyl acetate concentration technique and agar plate culture for diagnosis of human strongyloidiasis. Journal of Clinical Microbiology, 2005, 43:1932-1933. doi:10.1128/ JCM.43.4.1932-1933.2005 pmid:15815023
25. Page WA, Dempsey K, McCarthy JS. Utility of serological follow-up of chronic strongyloidiasis after anthelminthic chemotherapy. Transactions of the Royal Society of Tropical Medicine and Hygiene, 2006, 100:1056-1062. doi:10.1016/j. trstmh.2005.12.006 pmid:16551471

26. Kearns $T$ et al. Ivermectin mass drug administration program to treat endemic scabies and strongyloidiasis in a remote aboriginal community in northern Australia. Tropical Medicine \& International Health, 2011, 16 S1;198-199.

27. Palmer CS et al. The veterinary and public health significance of hookworm in dogs and cats in Australia and the status of A. ceylanicum. Veterinary Parasitology, 2007, 145:304-313. doi:10.1016/j.vetpar.2006.12.018 pmid:17276602

28. Koehler AV et al. Genetic characterization of selected gastrointestinal parasites associated with humans using a mutation scanningcoupled approach. Electrophoresis, 2013, 34:1720-1722. doi:10.1002/elps.201300100 pmid:23592267

29. Prociv P, Luke RA. Evidence for larval hypobiosis in Australian strains of Ancylostoma duodenale. Transactions of the Royal Society of Tropical Medicine and Hygiene, 1995, 89:379. doi:10.1016/0035-9203(95)90016-0 pmid:7570868

30. Zuk M, McKean KA. Sex differences in parasite infections: patterns and processes. International Journal for Parasitology, 1996, 26:1009-1023. doi:10.1016/S0020-7519(96)00086-0 pmid:8982783

31. Adams M, Page W, Speare R. Strongyloidiasis: an issue in Aboriginal communities. Rural and Remote Health, 2003, 3:152. pmid: 15877491

32. Prociv P. Zoonotic hookworm infections (ancylostomosis). In Palmer SR, Lord Soulsby, Simpson DIH, editors. Zoonoses: biology, clinical practice, and public health control. Oxford, Oxford University Press, 1998, p. 803-822.

33. Currie B, Anstey N. Eosinophilic enteritis in the Northern Territory. The Medical Journal of Australia, 1991, 154:71. pmid:1984596

34. Conner G. Diagnosis and epidemiology of Toxocara canis infection in humans (Masters of Applied Science thesis). Sydney, University of Technology, 1990.

35. Dreyer $\mathrm{G}$ et al. Patterns of detection of Strongyloides stercoralis in stool specimens: implications for diagnosis and clinical trials. Journal of Clinical Microbiology, 1996, 34:2569-2571. pmid:8880521

36. Davies J et al. Hookworm in the Northern Territory: down but not out. The Medical Journal of Australia, 2013, 198:278-281. doi:10.5694/mja12.11615 pmid:23496406

37. Kearns $T$ et al. Faecal parasitology of human specimens collected from a remote Aboriginal community in the Northern Territory. ASM/ACTM Poster abstract, Parasitology Masterclass 14-17 July, 2011, Cairns. Annals of the ACTM, 2011, 12:55.

38. Horton J. Albendazole: a review of anthelmintic efficacy and safety in humans. Parasitology, 2000, 121 Suppl;S113-132. doi: $10.1017 /$ S0031182000007290 pmid:11386684

39. Reynoldson JA et al. Efficacy of albendazole against Giardia and hookworm in a remote Aboriginal community in the north of Western Australia. Acta Tropica, 1998, 71:27-44. doi:10.1016/ S0001-706X(98)00048-5 pmid:9776141

40. Castles I. Census counts for small areas: Northern Territory 1991 Census of Population and Housing. Canberra, Australian Bureau of Statistics, 1993.

41. Li SQ et al. From infancy to young adulthood: health status in the Northern Territory. Darwin, Northern Territory Government Department of Health and Community Services, 2006. 